\title{
CartesI/O: A ROS Based Real-Time Capable Cartesian Control Framework
}

\author{
Arturo Laurenzi ${ }^{1,2}$, Enrico Mingo Hoffman ${ }^{1}$, Luca Muratore ${ }^{1,3}$, and Nikos G. Tsagarakis ${ }^{1}$
}

\begin{abstract}
This work introduces a framework for the Cartesian control of multi-legged, highly redundant robots. The proposed framework allows the untrained user to perform complex motion tasks with robotics platforms by leveraging a simple, auto-generated $R O S$-based interface. Contrary to other motion control frameworks (e.g. ROS MoveIt!), we focus on the execution of Cartesian trajectories that are specified online, rather than planned in advance, as it is the case, for instance, in tele-operation and locomotion tasks. Moreover, we address the problem of generating such motions within a hard realtime $(R T)$ control loop. Finally, we demonstrate the capabilities of our framework both on the COMAN+ humanoid robot, and on the hybrid wheeled-legged quadruped CENTAURO.
\end{abstract}

\section{INTRODUCTION}

In the past few decades, advancements in robotics have vastly extended its domain of application, moving from the traditional industrial focus to eventually applying robots to human and more unstructured environments. Consequently, robotic platforms have grown in complexity, in an attempt to satisfy the requirements that the new applications demand in terms of hardware, motion generation, control and human machine interfaces. Indeed, operating effectively in complex environments requires enhanced mobility in order to move on rough surfaces, overcome obstacles, and to carry out complex manipulation tasks that take place in an extended workspace, ranging from the ground-level to above the eye-level.

Such robots exhibit a high degree of redundancy, which however complicates their control from several perspectives: first, tasks have usually different priorities; then, when infinitely many solutions exist, some criterion is needed to select one. Finally, the complexity of the code required to control the robot grows considerably: a simple-yet-effective scheme for solving the manipulator inverse kinematics may be realized with few lines of code, whereas the control of highly redundant robots is best done inside a suitable framework that hides most of the involved complexity in the motion coordination of redundant robots.

An aspect that is worth highlighting is the dichotomy between tasks that are pre-planned offline, and tasks that are specified online, in a continuous fashion. As an example from the first family, the reader could consider a reachto-grasp task taking place in a cluttered environment; such an action can be completely pre-planned before the actual

\footnotetext{
${ }^{1}$ Advanced Robotics Department (ADVR), Istituto Italiano di Tecnologia, Genova, Italy

${ }^{2}$ DIBRIS, Università di Genova, Italy

${ }^{3}$ School of Electrical and Electronic Engineering, The University of Manchester, M13 9PL, UK

\{arturo.laurenzi, enrico.mingo, luca.muratore, nikos.tsagarakis\}@iit.it
}

execution, thus opening the possibility of performing a timeconsuming search over the robot configuration space in order to find obstacle-free trajectories. Conversely, examples from the second category include tele-operation scenarios, reactive locomotion and physical interaction, and others. Online tasks greatly differ from their pre-planned counterparts, since they require low computation times and, especially when any kind of feedback is involved, small delay and jitter in the execution.

Existing works have recognized the need for a Cartesian control framework; yet, it is hard to find a solution that satisfies the following requirements:

- flexible task specification, in terms of both type and number of tasks;

- ability to enforce soft priorities as well as hard priorities between tasks;

- ability to specify constraints in the task execution;

- small computation time (suitable for online execution);

- possibility to execute inside a real-time $(R T)$ thread in order to reduce delays and jitter;

- ease of configuration and use, quick setup time and ready to use control tools;

- parametrized with standard description formats (e.g. $U R D F$ ) in order to support multiple platforms;

- handling of floating base robots.

Previous work from the authors [1], [2] goes in the direction outlined by the aforementioned points, resulting in the $\mathrm{C}++$ library OpenSoT, which provides tools for writing Cartesian solvers while taking into account priorities and constraints, in a real-time safe way. In the present article, we extend the framework with additional layers that permit to perform Cartesian control "out of the box": we relieve the user from the need of writing and compiling $\mathrm{C}++$ code that is tailored to a specific robotic platform and task, and we provide an autogenerated uniform interface to send commands to Cartesian controllers inside the popular ROS framework. Notably, the same ROS API is provided even when Cartesian controllers run inside a hard real time thread.

We organize the rest of the article as follows: in Section II. we review some of the related works; then, in Section III the overall architecture of the proposed framework is described. Section IV is dedicated to the description of two experimental setups that validate the effectiveness of the framework. Finally, possible future directions are depicted in Section $\mathrm{V}$

\section{RELATED WORKS}

The presented framework draws inspiration from previous work on the same topic; in the remainder of this section 


\begin{tabular}{lcccc}
\hline & $\begin{array}{l}\text { Environment-aware } \\
\text { planning capabilities }\end{array}$ & Real time capable & Generic robot support & $\begin{array}{l}\text { Flexible task specifica- } \\
\text { tion }\end{array}$ \\
\hline OpenRAVE [3] & $\checkmark$ & $\checkmark$ & $\checkmark$ & $\boldsymbol{X}$ \\
MoveIt! [4] & $\checkmark$ & $\boldsymbol{x}$ & $\checkmark$ & $\boldsymbol{X}$ \\
Chorenoid [5] & $\boldsymbol{x}$ & $\checkmark$ & $\boldsymbol{X}$ & $\boldsymbol{X}$ \\
CartesI/O & $\boldsymbol{X}$ & $\checkmark$ & $\checkmark$ & $\checkmark$ \\
\hline
\end{tabular}

TABLE I

COMPARISON BETWEEN DIFFERENT FRAMEWOKS FOR ROBOT CARTESIAN CONTROL.

we present a summary of the most prominent contributions in the field, highlighting the shortcomings that we aim to address with our framework. A summary of our comparison is outlined in Table 1 .

OpenRAVE [3] is one of the first environments for testing, developing, and deploying motion planning algorithms in real-world robotics applications. It has been used with many different types of robots such as humanoids, mobile platforms and manipulators. From the Cartesian control point of view, OpenRAVE relies on ikfast which is an analytical IK engine; it is one of the fastest IK engines available at the moment, however it does not handle redundancy (extra DoFs have to be locked manually in order to match the required kinematic structure).

The most widely-spread framework for Cartesian control is probably MoveIt! [4], which is part of the open source ROS framework. MoveIt! provides a rather complete system of motion planning and execution tools that also take into account the perceived environment. The main core of MoveIt! is based on state-of-the-art sampling-based motion planning algorithms, mostly deployed inside the OMPL project [6]. Despite MoveIt! has been used on many different types of robot, it lacks explicit support for multi-chains and floatingbase (e.g. legged) robots. Furthermore its non-linear planning nature does not make it suitable for on-line (tele-operation) or real-time control.

Another notable work is the software suite Chorenoid [5], which permits to synthesize whole body motions for bipedal humanoid robots. The user can control different end-effectors as well as perform dynamic motions which are filtered through a Zero-Moment-Point (ZMP) based stabilizer. Such a tool has been used successfully on many HRP-series robots, yet it does not permit to setup customized IK problems. This indeed limits the usability of Chorenoid to manipulators and bipeds.

A lot of effort was spent on creating GUI systems for the DARPA Robotics Challenge (DRC) (e.g. [7], [8], [9]). Yet, none of these works was designed to be general enough to handle different types of robots with considerably diverse structure. Moreover, most of these GUI systems focus on predefined tasks (usually arms end-effectors, center-of-mass, and direct joint-space control) without any flexibility in terms of tasks or constraints declaration.

\section{ARCHITECTURE AND IMPLEMENTATION}

The purpose of this section is to present details about the framework design and structure. First and foremost, we highlight which are the main building blocks of the framework; then, we briefly introduce our previous work [1], the OpenSoT library. Finally, from Section III-C to Section III-E we present the core components of CartesI/O, that is the main focus of this work.

\section{A. Framework components}

Following a bottom-up description, we can distinguish:

- the solver; this is the component that solves a single instance of the mathematical problem that describes our Cartesian control algorithm. In our case, this could be either a matrix pseudo-inverse solver, or a Quadratic Program $(Q P)$ solver. Other possibilities exist, like more general Non-Linear Program (NLP) solvers.

- A modeling language that allows to construct the aforementioned mathematical problems in a natural and more high-level way, which is less error-prone and time consuming.

- A base class for Cartesian controllers, that allows for uniform programmatic usage of any specific implementation. It also takes care of set-point management, as for instance enforcing velocity/acceleration limits, or transforming waypoints into properly interpolated references.

- A middleware interface, which enables all other processes that compose the control system to send their references in a uniform way that does not depend on the specific implementation running.

This work is mostly concerned with the last two parts, and it is completely decoupled from any specific choice of a solver and modeling language. However, integration with the OpenSoT library, which implements a modeling language tailored to Cartesian control, also represents a major goal; we briefly describe OpenSoT in the following section.

\section{B. The OpenSoT library}

The OpenSoT library is a $\mathrm{C}++$ framework which has been initially developed to participate to the DARPA Robotics Challenge [10] as a whole-body inverse kinematics engine. During the years, inverse dynamics and force optimization have also been integrated [1], [2] for different robotic platforms. The focus of OpenSoT is to ease the formalization of prioritized controllers through dedicated interfaces for tasks, 
constraints and solvers. Each of these entities are atomic elements which can be combined using a simple syntax, named Math Of Task. An example of a simple controller described using the Math Of Task is the following one:

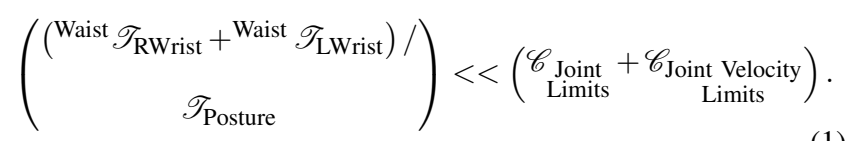

Two hard priorities (slash "/" operator) are specified: the first one is constituted by two tasks (in relative soft priority, plus "+" operator) which control the arms end-effectors w.r.t. the Waist frame, and the second one is a Joint Postural task. All priority levels are subject to Joint Limits and Joint Velocity Limits constraints (" $<<$ " operator).

The control problem (1) is then solved in two steps: first, a solver front-end computes matrices and vectors that describe the QP problems for all priority levels, according to the following formulation:

$$
\begin{gathered}
\min _{\boldsymbol{x}_{i}}\left\|A_{i} \boldsymbol{x}_{i}-b_{i}\right\|^{2}+\varepsilon\left\|\boldsymbol{x}_{i}\right\|^{2} \\
\text { s.t. } b_{1} \leq D \boldsymbol{x}_{i} \leq b_{\mathrm{u}} \\
u_{1} \leq \boldsymbol{x}_{i} \leq u_{\mathrm{u}} \\
A_{i-1} \boldsymbol{x}_{i-1}^{*}=A_{i-1} \boldsymbol{x}_{i} \\
\vdots \\
A_{0} \boldsymbol{x}_{0}^{*}=A_{0} \boldsymbol{x}_{i} .
\end{gathered}
$$

Then, a solver back-end ${ }^{1}$ actually computes the corresponding solutions. Notice that the Math Of Task formulation, as well as the chosen solver, are completely decoupled from the type of controller (velocity or acceleration-level IK, torque control, ...) which depends only on the specific tasks and constraints implementations.

\section{Cartesian Interface}

A modeling language eases the job of formulating and solving a complex optimization problem; yet, the need to write $\mathrm{C}++$ code that is customized for the specific robotic platform and task to be solved remains. This should be avoided, both with a view to promote code reuse, and also considering that, for complex platforms and according to the authors' experience, writing a hierarchy of tasks/constraints that makes the robot show the desired behavior can be "an art". Therefore, it should be left to "experts in the field", while users should simply customize the problem to better fit their needs. Furthermore, this code would be of little use without an I/O infrastructure that allows a control module to communicate with the external world. Again, the user should be relieved from developing its own. With this motivation, we started developing an interface layer that:

- provides a uniform way to programmatically interact with a Cartesian controller;

- automatically generates a complete ROS API for sending references to the controller;

- allows to use the ROS-based API also in the case that the solver is running inside a real-time thread.

\footnotetext{
${ }^{1}$ At this moment, back-ends are available for the qpOASES [11] and $O S Q P$ [12] solvers.
}

We call the base class that specifies such an interface CartesianInterface. It defines simple methods that can be used to change the behavior of all defined tasks, as for instance:

- a setPoseReference() method sets the Cartesian set-point corresponding to a task, given its controlled link name;

- the corresponding setWayPoints() method is used to specify a point-to-point motion passing through custom waypoints.

- A setBaseLink() method can be used to change online the base-link of a task.

- A setControlMode() method is used for selecting whether a specific task should be position-controlled, velocity-controlled, or disabled at all. Tasks that are running in velocity mode will discard any position reference, while tasks that are disabled will disappear completely from the Cartesian control problem.

- An update() method is used to evaluate all point-topoint trajectories given the current time, and to enforce velocity/acceleration limits by means of the Reflexxes library [13]. This method is overridden by the specific subclasses in order to implement their own control loop.

The developer willing to implement its own Cartesian controller can override all these methods in order to take appropriate actions whenever a reference, a control mode or a base-link has changed.

To store the state of the robot, and to perform kinematic/dynamic computation as well, we make use of the XBot::ModelInterface class from the XBotCore framework [14], [15], which acts as a wrapper for rigid body dynamics libraries. Specific implementations of it can be chosen at runtime, using a dynamic loading mechanism.

\section{Auto-generated ROS API}

Once that a Cartesian controller has been implemented, a communication layer towards external modules is needed, which is our middleware interface. This is implemented in the form of a $\mathrm{C}++$ class that we call ROS Server Class; for each of the defined tasks, it provides the following functionalities:

- a TF publisher for the model state;

- point-to-point motions with custom waypoints through a custom ROS action;

- commanding continuous pose and velocity references by publishing to ROS topics;

- run-time activation/deactivation of tasks, as well as change of control mode and base-link by calling custom ROS services;

- Rviz interactive markers manager [18] that allows for intuitive, GUI-based reference generation;

- joystick-based reference generation;

- joint sliders, which permit to work at joint space level.

2 An RBDL-based [16] version is available at https://github. com/ADVRHumanoids/ModelinterfaceRBDL, a version based on iDynTree [17] is available at https://gitlab.advrcloud.iit. it/advr_humanoids/modelinterfaceidynutils 


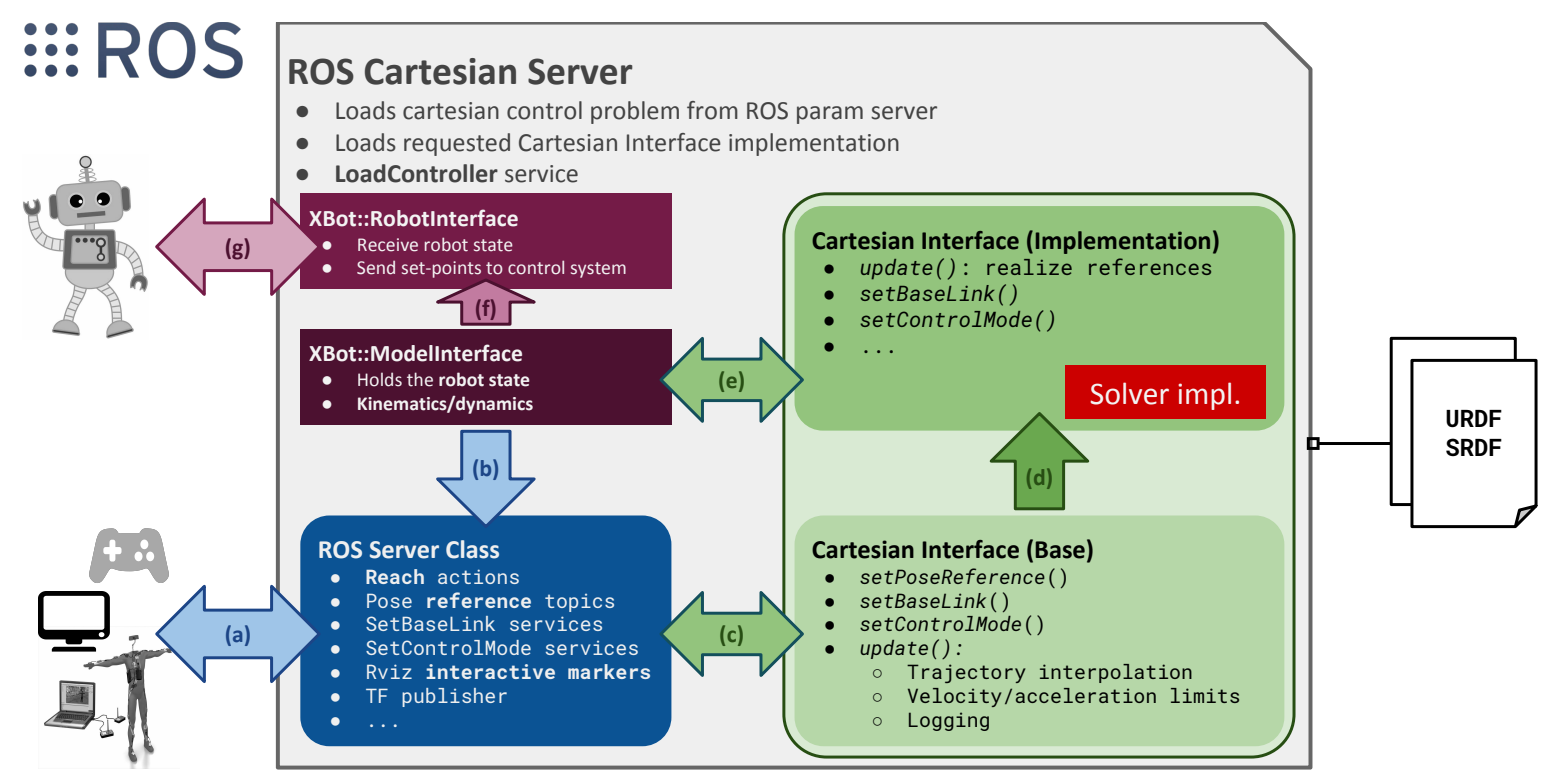

Fig. 1. Components of the Cartesian Interface and signal flow when the Cartesian Interface is executed inside a ROS node. External processes exchange information with the CartesI/O framework via ROS topics (a), thanks to the ROS Server Class component. It gets the current solver state through the ModelInterface object (b), in order to broadcast it to the ROS environment. It also forwards the received commands to the Cartesian Interface base class (c), where trajectory interpolation and filtering take place. The Cartesian Interface implementation component gets these pre-processed signals in order to track them (d). This results in an updated model state (e), which is then sent to the robot actuators through the RobotInterface object (f, g).

Furthermore, a ROS node that acts as a Cartesian Server is provided as well. It loads a user-specified implementation of the CartesianInterface class using a dynamic loading mechanism, and initialize the ROS Server Class in order to generate the ROS API. Finally, a ROS service is provided to dynamically change the controller that is under execution during runtime. The resulting architecture is shown in Figure 11. note that, in order to send actual references to the robot, we employ the XBot::RobotInterface class from the XBotCore framework, which serves as the robot abstraction layer ${ }^{3}$

\section{E. Configurable OpenSoT implementation}

As the final step towards a complete Cartesian control framework as described in Section III-A we provide a generic implementation of the CartesianInterface that relies on OpenSoT as the modeling language, and on its supported solvers for carrying out the actual optimization procedure. Such a module allows the user to formulate a hierarchical, whole-body inverse kinematics problem at the velocity level (as described in Section [III-B); it is written so as to be completely configurable, meaning that the stack of tasks can be specified either on a YAML file or via the ROS parameter server. In this way, starting from a standard description of the robot in terms of its URDF/SRDF, the user can directly run a ROS Cartesian Server to perform rather complex whole-body control tasks with no code compilation involved; moreover, the auto-generated ROS API provides a convenient way

3 A ROS-based version that eventually publishes on a joint state topic is available at https://github.com/ADVRHumanoids/ Robot InterfaceROS to interact with such a controller from all processes that compose a distributed control system.

\section{F. Real-time execution}

Whenever a Cartesian controller is based on continuous feedback from the robot, its precise and jitter-free execution at the specified control frequency becomes critical; indeed, delays contribute to destabilize feedback loops, and should therefore be avoided. Typical examples are torque-based controllers (e.g. Cartesian impedance control); however, it is worth noticing that position-based controllers can involve feedback, as in the case of admittance controllers, stabilizers for legged robots (e.g. [19]), and tele-manipulation with force feedback.

Such characteristics can be achieved by calling the CartesianInterface's update() method from within a real-time $(R T)$ thread that runs inside a suitable real-time operating system (RTOS); in addition, the communication between this thread and the robot control PC needs to be fast introducing minimum latency. In order to do so, we need to ensure realtime safeness of all components of our architecture, which broadly speaking means that all non-deterministic operations should be avoided, most notably memory allocations and, e.g., network communication.

By careful code development and profiling, we ensure satisfaction of these constraints both by the OpenSoT library (including its solver back-ends), and by the CartesianInterface layer. However, it is not possible to run our ROS Server Class on the RT layer directly, mainly because of ROS's usage of TCP primitives 4 To address this issue, a dual-thread

\footnotetext{
${ }^{4} \mathrm{TCP}$ usage from a real time thread will eventually will be possible in ROS2, which is based on the DDS middleware.
} 
architecture is needed, where a non-RT thread runs our ROS API server, while a $R T$ thread runs the CartesianInterface implementation. We put this idea into practice by leveraging the XBotCore framework, that provides us both a RT "control thread" and a non-RT "communication thread". We design the synchronization between the two to be lock-free for the RT thread, in order to avoid priority inversion problems. This results in a deterministic execution time for our controller, as we experimentally demonstrate in Section IV-A

\section{EXPERIMENTAL RESULTS}

To validate our CartesI/O framework and demonstrate its flexibility in different robot platforms, we set up two manipulation tasks to be carried out by our legged robots CENTAURO [20], a 39-DoF wheeled-legged quadruped with a humanoid torso, and the 28-DoF humanoid COMAN+. In both cases, we select a box-picking task where the box must be picked from a low height. In such a case, not only the arms but all the robot chains must coordinate to accomplish the task, which highlights the advantage of using a floating-base whole-body formulation. The outcome of our experiments is summarized in the accompanying video; an extended version can be found at https: / / youtu.be/eVmDBVL83WY.

\section{A. Case study: stabilized box-picking task}

As our first experiment, we present an application of our framework to a scenario where the humanoid COMAN+ has to pick a $3 \mathrm{Kg}$ box and pass it to a human. We define the task such that the robot must reject external disturbances without falling, and it must also show compliance in the reaction in order not to hurt people around it. To achieve compliant rejection of external forces, we use the work of [19] where a compliant admittance-base stabilizer is introduced, which essentially computes a modified CoM reference from a reference on the center of pressure $(\mathrm{CoP})$ (which we kept fixed during the experiment) and from force-torque measurements at the feet as well. Since this experiment involves a feedback controller, we run our Cartesian solver plus the stabilizer from within the XBotCore RT control thread as explained in Section III-F. To execute the motions, we run from a different low-priority process a $R O S S M A C H$-based state machine written in Python that sends target poses to the end-effectors via our auto-generated ROS API as follows:

- the box is grasped and brought to the chest level by sending suitable references to the hands w.r.t. the world frame;

- the box is then passed to the human operator; in order to do so, we change the base link for the hands tasks from the world frame to the torso frame, and finally we command it to rotate about the $z$-axis.

During the whole demo, the compliant stabilizer is continuously adjusting the CoM reference to track the desired CoP.

Time statistics regarding the experiment are shown in Figure 2, it can be noticed that our control thread does indeed

5 http://wiki.ros.org/smach

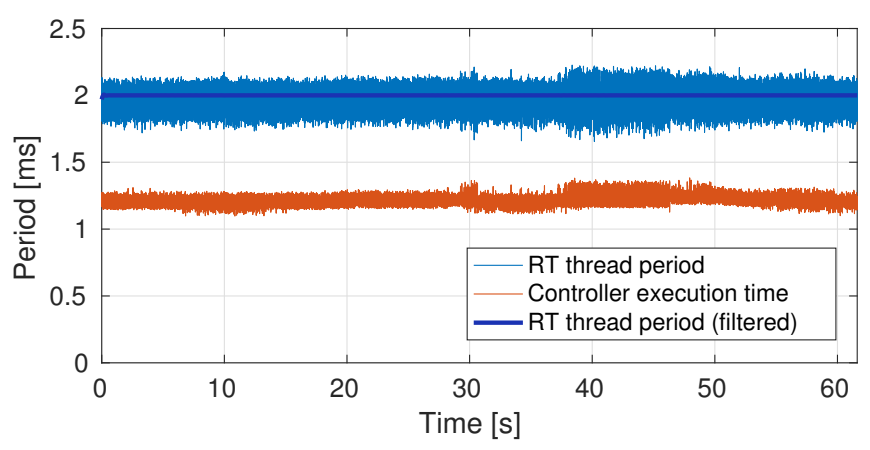

Fig. 2. Timing statistics for the box picking experiment with COMAN+ The thin blue line represents the XBotCore RT thread period. The cartesian controller computation time (including the stabilizer) is depicted in red.

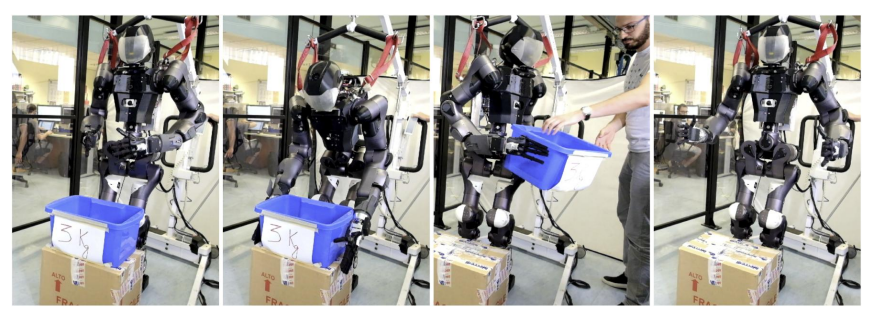

Fig. 3. Snapshots from a box-picking task with RT stabilization using humanoid COMAN+.

meet its deadline in a deterministic way, with a root-meansquare deviation of $T_{\mathrm{jitter}} \approx 90 \mu \mathrm{s}$, over more than one minute of experiment. Snapshots from the experiment are visible in Figure 3 .

\section{B. Case study: ground-level bimanual manipulation}

For our second experiment, we choose to pick up a $6 \mathrm{~kg}$ brick from the ground using the CENTAURO robot, and then pass it to a human operator. Since the operator is standing on one side of the robot, the robot must perform a brief wheeled-locomotion phase in order to turn ninety-degrees. This is done by switching at runtime between two different implementation of the Cartesian Interface: the first, which is used to pick up the box, is the dynamically-configurable IK controller described in Section III-E, the second one, which we call Centauro Wheeled Motion, is an IK controller that is tailored to the mixed wheeled-legged locomotion of the CENTAURO robot. It is implemented by means of the OpenSoT library, and it permits to:

- control the waist pose w.r.t. the world through appropriate steering/rolling of the wheels;

- control the wheels position w.r.t. the waist frame in order to adjust the support polygon shape;

- perform basic control of the end-effectors w.r.t. waist frame.

Since this demonstration does not require to meed hard deadlines, we run the Cartesian controllers on the ROS Cartesian Server at a frequency of $100 \mathrm{~Hz}$. Again we script the robot behavior via a $R O S S M A C H$ state machine as follows: 


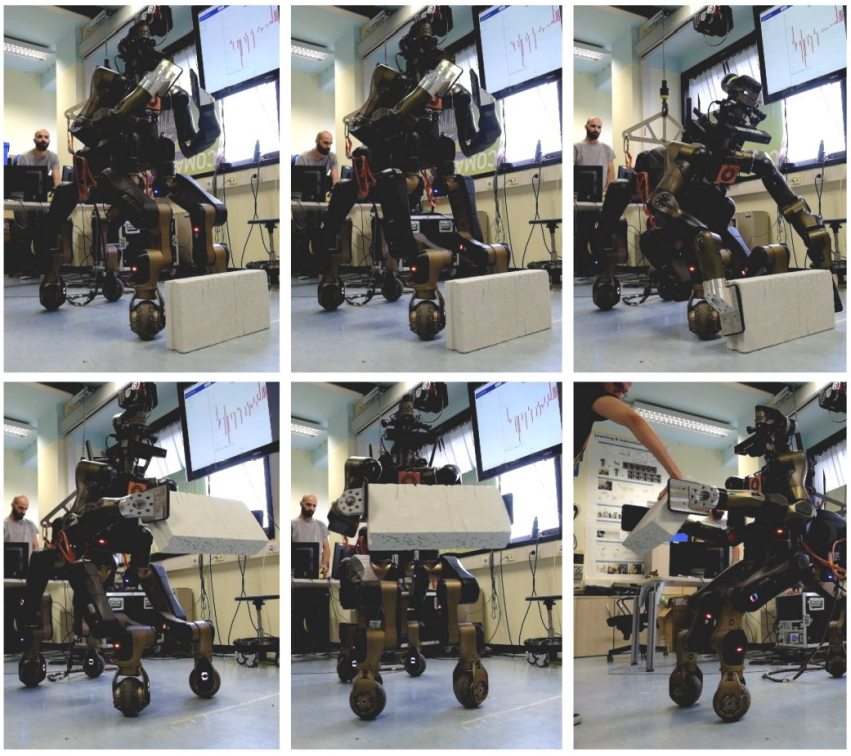

Fig. 4. Snapshots from the experiment with CENTAURO robot described in Section IV-B

- first, we change the postural for the front knees in order to avoid later collisions with the arms;

- then we command the end-effector to surround the box, then grasp it and bring it up;

- after this, we load our Centauro Wheeled Motion, and we command a ninety-degrees rotation to the waist;

- finally, we command the arms to open in order to release the box.

The outcome of this experiment is summarized in Figure 4. and in the accompanying video as well.

\section{CONCLUSiONS}

In this work we have introduced CartesI/O, a framework for Cartesian control of floating/fixed-base robots which is focused on online execution, possibly under real-time constraints. As our main contribution, we integrate Cartesian solvers inside an architecture that permit to interact with them in a uniform way. This is achieved at two different levels:

- programmatically, through our CartesianInterface base class;

- from the ROS middleware, via an auto-generated set of topics, services, actions and tools that can be used to monitor the solver state, send references, and customize the solver behavior.

Our API aims at providing highly flexible task customization during runtime: the user can activate/deactivate tasks, change their base-link, switch between position and velocity control, and even dynamically load different controllers. Furthermore, the framework integrates common tools, such as trajectory interpolation with way-points, as well as enforcement of user-specified velocity/acceleration limits.

A configurable inverse-kinematics solver is also provided, through which the user can quickly set up a Cartesian control problem from a YAML file, which is then solved using the OpenSoT library. Such a controller is designed to be real-time safe, allowing for the deterministic execution of the commanded references. Moreover, we provide tools to connect a RT Cartesian controller to our auto-generated ROS API, in order to enable mixed RT/non-RT robot control.

Future work will address the development of solvers working at the dynamics level, as well as the augmentation of the markers capabilities (e.g. Cartesian impedance markers). Moreover, we aim to add previewing capabilities, and environment-aware trajectory planning, e.g. via integration with MoveIt!. Finally, we aim at integrating this work with well-known RT frameworks such as OROCOS and ROS2.

\section{ACKNOWLEDGMENTS}

The research leading to these results has received funding from the European Union's Horizon 2020 research and innovation programme under grant agreements No. 644839 (CENTAURO) and No. 644727 (CogIMon).

\section{REFERENCES}

[1] E. M. Hoffman, A. Rocchi, A. Laurenzi, and N. G. Tsagarakis, "Robot control for dummies: Insights and examples using opensot," in 17th IEEE-RAS International Conference on Humanoid Robotics, 2017, pp. 736-741, 2017.

[2] E. M. Hoffman, A. Laurenzi, L. Muratore, D. G. Caldwell, and N. G. Tsagarakis, "Multi-priority cartesian impedance control based on quadratic programming optimization," in 2018 IEEE International Conference on Robotics and Automation (ICRA), 2018.

[3] R. Diankov, Automated Construction of Robotic Manipulation Programs. PhD thesis, Carnegie Mellon University, Robotics Institute, August 2010.

[4] S. Chitta, I. Sucan, and S. Cousins, "Moveit!," IEEE Robotics \& Automation Magazine, vol. 19, no. 1, pp. 18-19, 2012.

[5] S. Nakaoka, S. Kajita, and K. Yokoi, "Intuitive and flexible user interface for creating whole body motions of biped humanoid robots," in Intelligent Robots and Systems (IROS), 2010 IEEE/RSJ International Conference on, pp. 1675-1682, IEEE, 2010.

[6] I. A. Şucan, M. Moll, and L. E. Kavraki, "The Open Motion Planning Library," IEEE Robotics \& Automation Magazine, vol. 19, pp. 72-82, December 2012. http: // ompl.kavrakilab.org

[7] S. Caron and Y. Nakamura, "Teleoperation system design of valve turning motions in degraded communication conditions," The 33-rd Annual Conference of the RSJ, 2015.

[8] M. Schwarz, T. Rodehutskors, et al., "Nimbro rescue: Solving disasterresponse tasks with the mobile manipulation robot momaro," Journal of Field Robotics, vol. 34, no. 2, pp. 400-425, 2016.

[9] M. Fallon, S. Kuindersma, S. Karumanchi, M. Antone, T. Schneider, H. Dai, C. P. D’Arpino, R. Deits, M. DiCicco, D. Fourie, et al., "An architecture for online affordance-based perception and whole-body planning," Journal of Field Robotics, vol. 32, no. 2, pp. 229-254, 2015.

[10] N. G. Tsagarakis, D. G. Caldwell, et al., "Walk-man: A highperformance humanoid platform for realistic environments," Journal of Field Robotics, vol. 34, no. 7, pp. 1225-1259, 2017.

[11] H. J. Ferreau, C. Kirches, A. Potschka, H. G. Bock, and M. Diehl, "Qpoases: a parametric active-set algorithm for quadratic programming," Mathematical Programming Computation, vol. 6, pp. 327-363, Dec 2014.

[12] B. Stellato, G. Banjac, P. Goulart, A. Bemporad, and S. Boyd, "OSQP: An operator splitting solver for quadratic programs," ArXiv e-prints, Nov. 2017.

[13] T. Kröger, "Opening the door to new sensor-based robot applications-the reflexxes motion libraries," in Robotics and Automation (ICRA), 2011 IEEE International Conference on, pp. 1-4, IEEE, 2011.

[14] L. Muratore, A. Laurenzi, E. M. Hoffman, A. Rocchi, D. G. Caldwell, and N. G. Tsagarakis, "Xbotcore: A real-time cross-robot software platform," in 2017 First IEEE International Conference on Robotic Computing (IRC), pp. 77-80, April 2017. 
[15] "XBotCore GitHub repository." https://github.com/ ADVRHumanoids/XBotCore/wiki/XBotInterface

[16] M. L. Felis, "Rbdl: an efficient rigid-body dynamics library using recursive algorithms," Autonomous Robots, pp. 1-17, 2016.

[17] F. Nori, S. Traversaro, J. Eljaik, F. Romano, A. Del Prete, and D. Pucci, "icub whole-body control through force regulation on rigid noncoplanar contacts," Frontiers in Robotics and AI, vol. 2, no. 6, 2015.

[18] D. Gossow, A. Leeper, D. Hershberger, and M. T. Ciocarlie, "Interactive markers: 3-d user interfaces for ros applications [ros topics]," IEEE Robot. Automat. Mag., vol. 18, pp. 14-15, 2011.

[19] C. Zhou, Z. Li, X. Wang, N. Tsagarakis, and D. Caldwell, "Stabilization of bipedal walking based on compliance control," Autonomous Robots, vol. 40, pp. 1041-1057, Aug 2016.

[20] L. Baccelliere et al., "Development of a human size and strength compliant bi-manual platform for realistic heavy manipulation tasks," in Intelligent Robots and Systems (IROS), 2017 IEEE/RSJ International Conference on, pp. 5594-5601, IEEE, 2017. 